\title{
Anatomie des Wissens
}

\author{
WISSENSCHAFT ERKLÄRT: AUFBAU WISSENSCHAFTLICHER ARBEITEN Das Lesen \\ wissenschaftlicher Artikel ist einfacher, als es auf den ersten Blick scheint. \\ Roger Hilfiker zeigt Ihnen, wie Sie sich in einem solchen Artikel zurechtfinden und \\ schnell die Informationen finden, die Sie suchen.
}

P

hysiotherapeuten müssen Nachweise

für die Wirksamkeit ihrer Behandlung erbringen, um diese belegen zu können. Da bleibt das Lesen von und das Auseinandersetzen mit wissenschaftlichen Arbeiten nicht aus. Doch häufig fehlt die Zeit, den Artikel vollständig zu lesen, obwohl man nur nach bestimmten Informationen sucht. Um diese zu finden, hilft es zu wissen, an welcher Stelle man sie suchen muss. Die gute Nachricht ist: Die Stellen sind in fast allen Studien dieselben.

Gegliedert mit IMRAD > Wer Abkürzungen mag, kann sich Folgendes merken: IMRAD. I steht für Introduction, M für Method, R für Results, A steht für nichts, höchstens für And - denn IMRAD klingt besser als IMRD -, und D steht für Discussion. So lauten in der Regel die Überschriften der einzelnen Abschnitte wissenschaftlicher Artikel.

Ein Artikel beginnt immer mit einer Zusammenfassung, auch Abstract genannt, die man im Internet oder auf Datenbanken meistens gratis lesen kann und die in der Regel die wichtigsten Informationen enthält. Im Anschluss folgt die Einleitung (engl.: Introduction), manchmal auch Background genannt. Hier steht, was durch diese Forschungsarbeit verändert werden soll oder warum die Untersuchung wichtig ist. Die letzten Sätze der Einleitung sind besonders interessant, da die Forscher dort noch einmal konkret erwähnen, welches Ziel sie mit der Studie verfolgen.

Intervention und Patienten kennenlernen > Im Anschluss daran folgt der Methodenteil (Methods). Dort beschreibt der Autor, was er gemacht hat, und liefert Informationen über die untersuchte Patientengruppe, die manchmal auch Subjects genannt wird. Außerdem beschreibt er die Studienmethode und die Intervention, optimalerweise so, dass sich

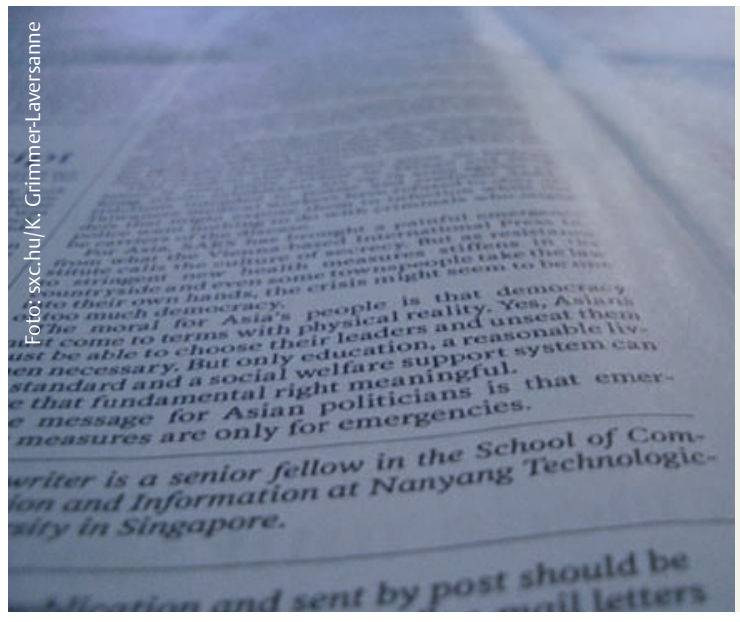

Wissenschaftliche Artikel sind gar nicht so unüberwindbar, wie sie auf den ersten Blick erscheinen. Denn: Sie folgen alle demselben Aufbau.

der Leser genau vorstellen kann, welche Behandlung die Probanden erhielten. Anschließend folgt eine Beschreibung der benutzten Tests und Apparate sowie der statistischen Analyse, die jedoch für Ungeübte oft leider nicht verständlich ist. Für andere Forscher ist dieser Teil der Arbeit jedoch wichtig, um die Studie wiederholen zu können.

Danach kommen die Resultate (Results), einerseits dargestellt in Grafiken und Tabellen, andererseits zusammengefasst im Text. Meist beschreiben die Autoren in der ersten Tabelle die Stichprobe (॰ physiopraxis 9/09, S. 22, „Wissenschaft erklärt: Die Stichprobe“), bevor sie in den nachfolgenden Tabellen oder Grafiken die Statistik oder andere Formen von Resultaten präsentieren.

Ergebnisse abwägen > Im letzten Teil des Artikels werden die Ergebnisse diskutiert (Discussion). Dieser Abschnitt beginnt, indem die Forscher noch einmal zusammenfassen, was sie gemacht und gefunden haben. Sie heben hervor, was diese Studie auszeichnet, stellen ihre Studie anderen Arbeiten mit ähnlicher Fragestellung gegenüber und erklären die
Gründe für etwaige Gemeinsamkeiten oder Unterschiede. Anschließend findet man unter dem Stichwort Limitations die Schwächen der Studie, zum Beispiel eine geringe Gruppengröße oder eine fehlende Verblindung der Probanden, und welchen Einfluss diese Einschränkungen auf die Resultate haben könnten.

Am Ende heben die Autoren hervor, was diese Resultate für die Forschung bedeuten, und man erfährt, ob weitere Studien nötig sind, um die gefundenen Ergebnisse zu bestätigen. Außerdem geben sie Hinweise, was man in künftigen Studien verbessern könnte und welche neuen Fragen sich aus den Ergebnissen ergeben haben.

Der ähnliche Aufbau von wissenschaftlichen Artikeln ist ein großer Vorteil. Er bietet dem Leser Orientierung auf dem abstrakten Gebiet der Forschung und erleichtert inm damit das Lesen von und das Arbeiten mit wissenschaftlichen Artikeln. Roger Hilfiker

\footnotetext{
$\Rightarrow$ Alle Artikel der Rubrik „Wissenschaft erklärt" finden Sie unter www.thieme.de/ physioonline > "physioexklusiv“ > "Wissenschaft erklärt“.
} 